\title{
Roles of Anion Sites in High-performance GeTe Thermoelectrics
}

\author{
Meng Li \\ University of Queensland \\ Shengduo Xu \\ University of Queensland \\ Min Hong \\ University of Southern Queensland \\ Wan-Yu Lyu \\ University of Southern Queensland

\section{Yuan Wang} \\ University of Queensland \\ Matthew Dargusch \\ The University of Queensland \\ Jin Zou
}

The University of Queensland https://orcid.org/0000-0001-9435-8043

Hui-Ming Cheng

Chinese Academy of Sciences

Zhi-Gang Chen ( $\square$ zhigang.chen@qut.edu.au )

Queensland University of Technology https://orcid.org/0000-0002-9309-7993

\section{Article}

Keywords:

Posted Date: January 21st, 2022

DOI: https://doi.org/10.21203/rs.3.rs-1237133/v1

License: @ (i) This work is licensed under a Creative Commons Attribution 4.0 International License.

Read Full License 


\title{
Roles of Anion Sites in High-performance GeTe Thermoelectrics
}

\author{
Meng $\mathrm{Li}^{1}$, Sheng-Duo $\mathrm{Xu}^{1}$, Min Hong ${ }^{2}$, Wan-Yu Lyu ${ }^{2}$, Yuan Wang ${ }^{1}$, Matthew \\ Dargusch $^{1}$, Jin Zou ${ }^{1,3}$, Hui-Ming Cheng ${ }^{4,5}$ and Zhi-Gang Chen ${ }^{1,6, *}$
}

${ }^{1}$ School of Mechanical and Mining Engineering, The University of Queensland; Brisbane, Queensland 4072, Australia.

${ }^{2}$ Centre for Future Materials, University of Southern Queensland; Springfield Central, Queensland 4300, Australia.

${ }^{3}$ Centre for Microscopy and Microanalysis, The University of Queensland; Brisbane, Queensland 4072, Australia.

${ }^{4}$ Shenyang National Laboratory for Materials Science, Institute of Metal Research, Chinese Academy of Sciences; Shenyang, 110016, P.R. China.

${ }^{5}$ Shenzhen Institute of Advanced Technology, Chinese Academy of Sciences; Shenzhen, 518055, P.R. China.

${ }^{6}$ School of Chemistry and Physics, Queensland University of Technology; Brisbane, Queensland 4072, Australia.

${ }^{*}$ Corresponding author. Email: zhigang.chen@qut.edu.au 


\begin{abstract}
Cation doping has been mainly used to enhance the performance of rocksalt GeTe thermoelectric materials. However, its counterpart, anion doping, is still in infancy. Here, we provide a theoretical insight into modifying the anion sites of GeTe for obtaining a better thermoelectric performance, envisaging its coordinate-based bonding mechanisms accounting for carrier and phonon transport characteristics. To support our point, we experimentally synthesized anion I doped GeTe samples, which show comparably optimized electrical and thermal properties compared to $\mathrm{Sb}$ or $\mathrm{Bi}$ doped GeTe samples. The (Bi, I) co-doped GeTe samples further obtain an enhanced figure-of-merit from 0.8 to 2.5 at $675 \mathrm{~K}$, which can be assembled as an 8-couple thermoelectric generator to yield a conversion efficiency of $\sim 11.6 \%$ under a temperature difference of $500 \mathrm{~K}$. This work rationalizes the potential of anion doping for high-performance GeTe thermoelectrics.
\end{abstract}


Thermoelectrics have attracted increasing interests from both academia and industries to alleviate the ever-growing energy crisis and emerging climate concerns. A typical thermoelectric generator can convert waste heat into electricity, with its efficiency determined by the heat dissipation from a nonequilibrium system. According to the Onsager's reciprocal relations, a high efficiency requires a combined large potential difference, low Joule heat, and sustained temperature gradient ${ }^{1}$. These requirements are corresponding to Seebeck coefficient $(S)$, electrical conductivity $(\sigma)$, and thermal conductivity $(\kappa)$ of a thermoelectric material, then the figure-of-merit,

$$
z T=S^{2} \sigma T / \kappa
$$

is defined to evaluate its goodness. In most cases, $\kappa$ can be written as a sum of electronic $\left(\kappa_{\mathrm{e}}\right)$ and lattice $\left(\kappa_{\mathrm{l}}\right)$ components, where $\kappa_{\mathrm{e}}$ is competing with power factor $\left(S^{2} \sigma\right)$ on carrier concentration $(n$, hole for $\mathrm{p}$ - and electron for n-type conductance), making a compromise needed for enhancing $z T^{2}$. In contrast, $\kappa_{1}$ is mainly determined by lattice vibration that can be quantified by phonons, and can therefore be minimized via both intrinsic (e.g., tuning phonon modes) and extrinsic (e.g., inducing scattering centers) processes ${ }^{3}$.

In addition to $z T$, a proper working temperature is important for the application of thermoelectric materials. For example, $\mathrm{Bi}_{2} \mathrm{Te}_{3}$ and $\mathrm{Mg}_{2} \mathrm{Bi} 3$ mainly work near room temperature, while half-heusler alloys are suitable for high temperatures ${ }^{4}$. For a mid-temperature range from 500 to $800 \mathrm{~K}$, MTe $(\mathrm{M}=\mathrm{Ge}, \mathrm{Sn}$, and $\mathrm{Pb})$ thermoelectric materials are mass-market candidates due to their high intrinsic $z T^{5}$. Other considerations are that PbTe is highly toxic, and SnTe suffers from its low $S$, making GeTe the most promising ${ }^{6}$. Fig. 1a summarizes recently reported MTe thermoelectric materials, which implies that optimizing $n$, i.e., by modulating reduced chemical potential $\left(u^{\mathrm{r}}\right)$, is effective for enhancing $z T$. Once an optimal $n$ is attained, the highest achievable $z T$ is mainly proportional to the quality factor $(B)$, which is only determined by weighed mobility $\left(\mu_{\mathrm{w}}\right)^{7}$. Given the semiconductor nature of MTe, doping is a facile and effective method to modify both $u^{\mathrm{r}}$ and $\mu_{\mathrm{w}}$, as has been extensively reported in cation doped in GeTe, $\mathrm{SnTe}$, and PbTe. In contrast, unlike SnTe and PbTe, anion doped GeTe is rarely reported. 
Such a mismatch between cation and anion doped GeTe seems unreasonable, considering the great success of anion doping in enhancing the $z T$ of analogous $\mathrm{SnTe}$ and $\mathrm{PbTe}{ }^{8}$. Here, we propose that anion and cation sites play a similar role in GeTe thermoelectric materials, not only because anion and cation sites are equivalent in the crystal structure thus the bonding mechanisms of GeTe, but also because anion and cation doping can comparably optimize carrier and phonon transport, thus electrical and thermal properties. To support our point, we synthesized cation $\mathrm{Sb} / \mathrm{Bi}$ and anion $\mathrm{Br} / \mathrm{I}$ doped, and (Bi, I) co-doped GeTe samples, where $\mathrm{Sb} / \mathrm{Bi}$ doped and I doped GeTe show comparably optimized electrical and thermal properties, yet due to different doping effects. An enhanced $z T$ from 0.8 to 2.5 at $675 \mathrm{~K}$ is achieved by $8 \%(\mathrm{Bi}, \mathrm{I})$ co-doped GeTe, which is assembled into an 8 -couple thermoelectric generator to yield a conversion efficiency of $\sim 11.6 \%$ under a temperature difference of $500 \mathrm{~K}$. The concurrent high material and device performance reported by this work justifies that anion doping is promising for realizing high-performance GeTe thermoelectrics.

\section{Crystal Structure and Bonding Mechanisms}

Pure GeTe undergoes a ferroelectric phase transition from high-temperature cubic $(\beta, F m \overline{3} m)$ to lowtemperature rhombohedral $(\alpha, R 3 m)$ phase at $\sim 670 \mathrm{~K}$, via a 3D Peierls distortion along its diagonal directions ${ }^{9}$. As displayed in Fig. $1 \mathrm{~b}, \beta$-GeTe shares a similar rocksalt structure with $\mathrm{SnTe}$ and $\mathrm{PbTe}$, which is stacked by cation and anion sub-lattices along their [111] axes. In this regard, cation and anion sites are spatially equivalent: in real space, they can interchange via the phase shift of a "periodic function"; whereas in reciprocal space, they can be folded into an identical k-point via the point group operations (Note S1). This leads to exceptional low-symmetry points in the first Brillouin zone of a rocksalt structured $\beta$-GeTe, which produces additional vellaytronics to optimize $n$, while restricts intervalley scattering to retain $B$, being beneficial for a high intrinsic $z T^{10}$. 
a

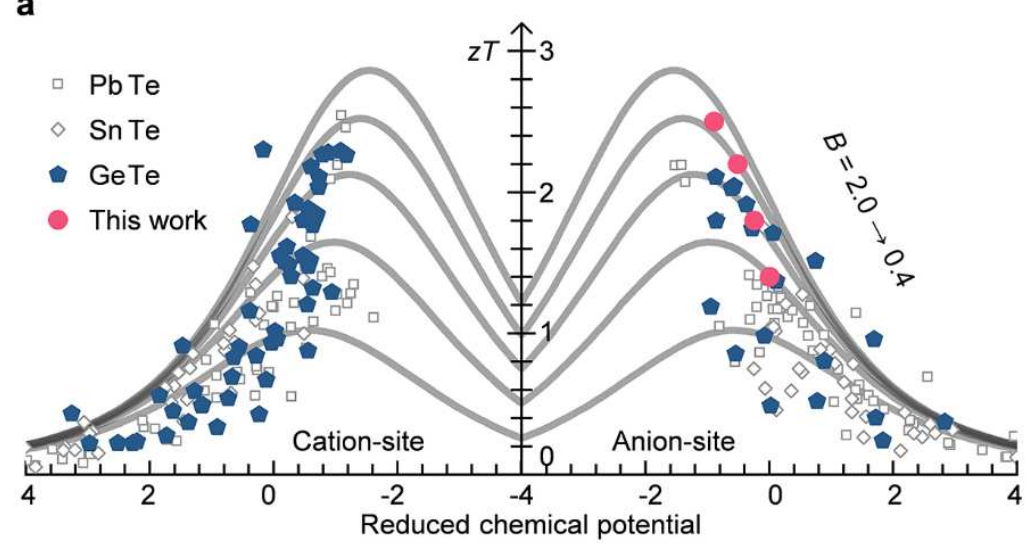

b
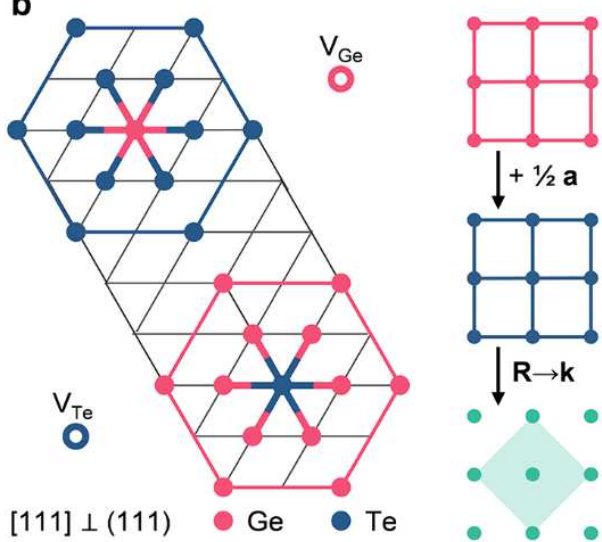

e

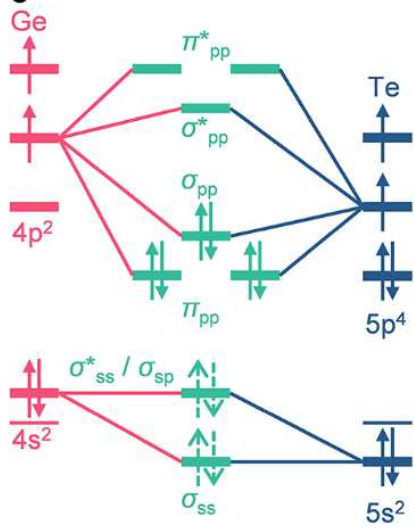

c

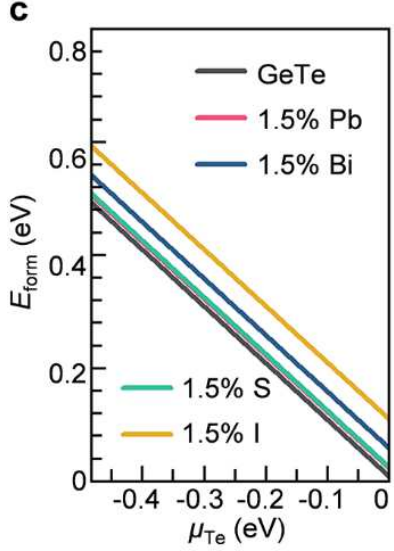

d

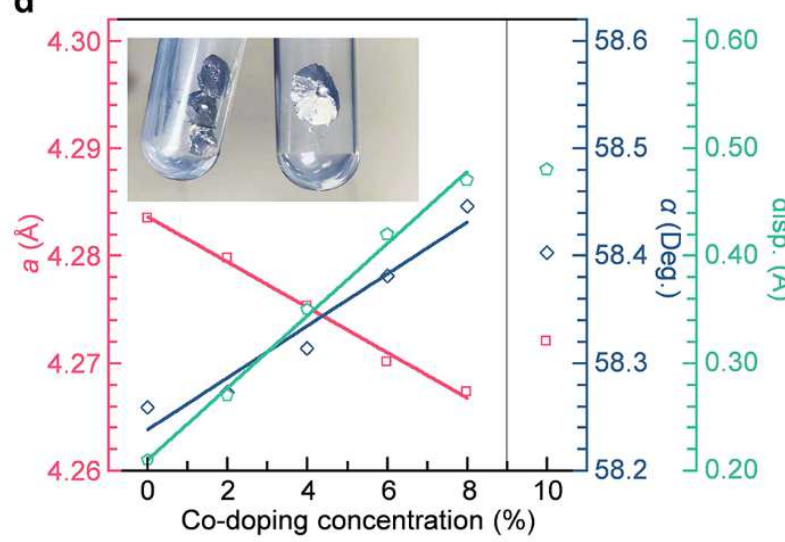

\section{.}


density-based properties of Ge-Te bonding in $\beta$-GeTe hasn't been fully understood, its much stronger covalence compared to $\mathrm{Na}-\mathrm{Cl}$ or $\mathrm{Zn}-\mathrm{Te}$ bonding is helpful for weakening the s-p hybridization to strengthen band anisotropy, which usually increases $S^{2} \sigma^{14}$. In addition, the long-range [001] polarity of Ge-Te bonding can intensify anharmonic phonon scattering and provide large phase space for three-phonon scattering processes, which decreases $\kappa_{1}{ }^{15}$.

However, a rocksalt coordinate can also be detrimental for the thermoelectric performance of $\beta$-GeTe. For instance, it is found that $\sim 3.5 \%$ Ge vacancies are intrinsically formed in $\beta$-GeTe due to low formation energy $\left(E_{\text {form }}\right)^{16}$. This exceeds $n$ beyond its optimal value, and degrades $z T$ from 1.7 in stoichiometric GeTe to less than 1.0 in Ge-deficient GeTe ${ }^{17}$. Fig. 1c calculates the $E_{\text {form }}$ of Ge vacancies in $\beta$-GeTe, which is elevated with respect to the chemical potential of anion Te $(u \mathrm{Te})$, therefore is more sensitive to anion doping. Specifically, doping $\sim 1.5 \% \mathrm{I}$ in GeTe $\left(\mathrm{Ge}_{64} \mathrm{Te}_{63} \mathrm{I}_{1}\right)$ increases the $E_{\text {form }}$ from $0.49 \mathrm{eV}$ in $\mathrm{Ge}_{64} \mathrm{Te}_{64}$ to $0.59 \mathrm{eV}$, which in turn decreases $n$ from $\sim 1 \times 10^{21}$ to $8.3 \times 10^{20} \mathrm{~cm}^{-3}$, towards the optimal value of $\sim 1 \times 10^{20} \mathrm{~cm}^{-3}$ (Note $\mathrm{S} 3$ ). To confirm this, we synthesized cation $\mathrm{Sb} / \mathrm{Bi}$ and anion $\mathrm{Br} / \mathrm{I}$ doped, and $(\mathrm{Bi}, \mathrm{I})$ co-doped GeTe samples by a modified flux-assist method (Note S4). The Rietveld refinement of X-ray diffraction (Fig. S2) confirms that the weight percentage of Ge impurities (due to Ge vacancies) is reduced from $2.7 \%$ in pure GeTe to $1.4 \%$ in $8 \%$ (Bi, I) co-doped GeTe. The lattice constant $(a)$, interaxial angle $(\alpha)$, and central site displacement (disp.) of matrix phase are variant according to (Bi, I) co-doping concentration, as plotted in Fig. 1d.

We then used electron microscopies to characterize the microstructures of I doped and (Bi, I) codoped GeTe. The fracture cross-sections observed using scanning electron microscopy (SEM, Fig. S3-5) indicates an apparent change of grain morphology, from nanometric flake grains in pure GeTe to micrometric polyhedron grains in $8 \%(\mathrm{Bi}, \mathrm{I})$ co-doped GeTe. This is consistent with the observed polished surface, consisted of nearly single crystal grains, in which a high density of twin structures with alternative contrast can be found. The twin structures are anisotropic along a set of orthogonal base zone axes, as revealed by the electron backscattered diffraction (EBSD, Fig. S6), with a 
misorientation angle between the twinned domains being either $88^{\circ}$ or $92^{\circ}$. Transmission electron microscopy (TEM) image further indicates a fractally second-order twin structures in a single twin domain (Fig. S7), elsewhere known as a herringbone structure ${ }^{18}$, which provides numerous twin boundaries and Ge plane vacancies, and therefore form ubiquitous lattice strain, devoting to an extrinsic process to decrease $\kappa 1^{19}$.

\section{Carrier and Phonon Transport}

The Ge-Te bonding in $\beta$-GeTe can be represented by a generalized energy level diagram for the interaction between cation $\mathrm{M}$ and anion X orbitals (Fig. S8). Specifically, $E_{\mathrm{M}}$ and $E_{\mathrm{X}}$ are on-site energies, which split into a bonding $\left(E_{\mathrm{MX}}\right)$ and an antibonding $\left.\left(E_{\mathrm{MX}}\right)\right)$ state. The difference from $E_{\mathrm{MX}}$ or $E_{\mathrm{MX}} *$ to the average of $E_{\mathrm{M}}+E_{\mathrm{X}}$ corresponds to the hypotenuse of a right-angled triangle with legs of energy gap ( $A$, governed by energy alignment) and overlap parameter ( $V$, governed by momentum alignment), given ${ }^{20}$ :

$$
E_{\mathrm{MX} / \mathrm{MX}^{*}}=1 / 2\left(E_{\mathrm{M}}+E_{\mathrm{X}}\right) \pm 1 / 2 \sqrt{\left(E_{\mathrm{M}}-E_{\mathrm{X}}\right)^{2}-4 V}
$$

Based on the tight binding model, Fig. 1e sketches the energy level diagram of overall chemical bonding in $\beta$-GeTe, as a linear combination of molecular orbitals ${ }^{21}$, where the $\mathrm{p}$-p bonding in higher energy than s-s bonding dominates carrier transport. To simplify analysis, the energy level is projected to a 1D diatomic chain from $\Gamma(0)$ to $L(\pi / a)$ point, i.e., along a [111] direction of $\beta$-GeTe, as shown in Fig. 2a. As $\pi$ type p-p $\left(\pi_{\mathrm{pp}}\right)$ bonding is symmetric along bonding axis, it has the lowest energy at $\Gamma(0)$ point, and an upward dispersion from $\Gamma$ to $L(\pi / a)$ point. In contrast, $\sigma_{\mathrm{pp}}$ bonding is antisymmetric along bonding axis with a downward dispersion. 

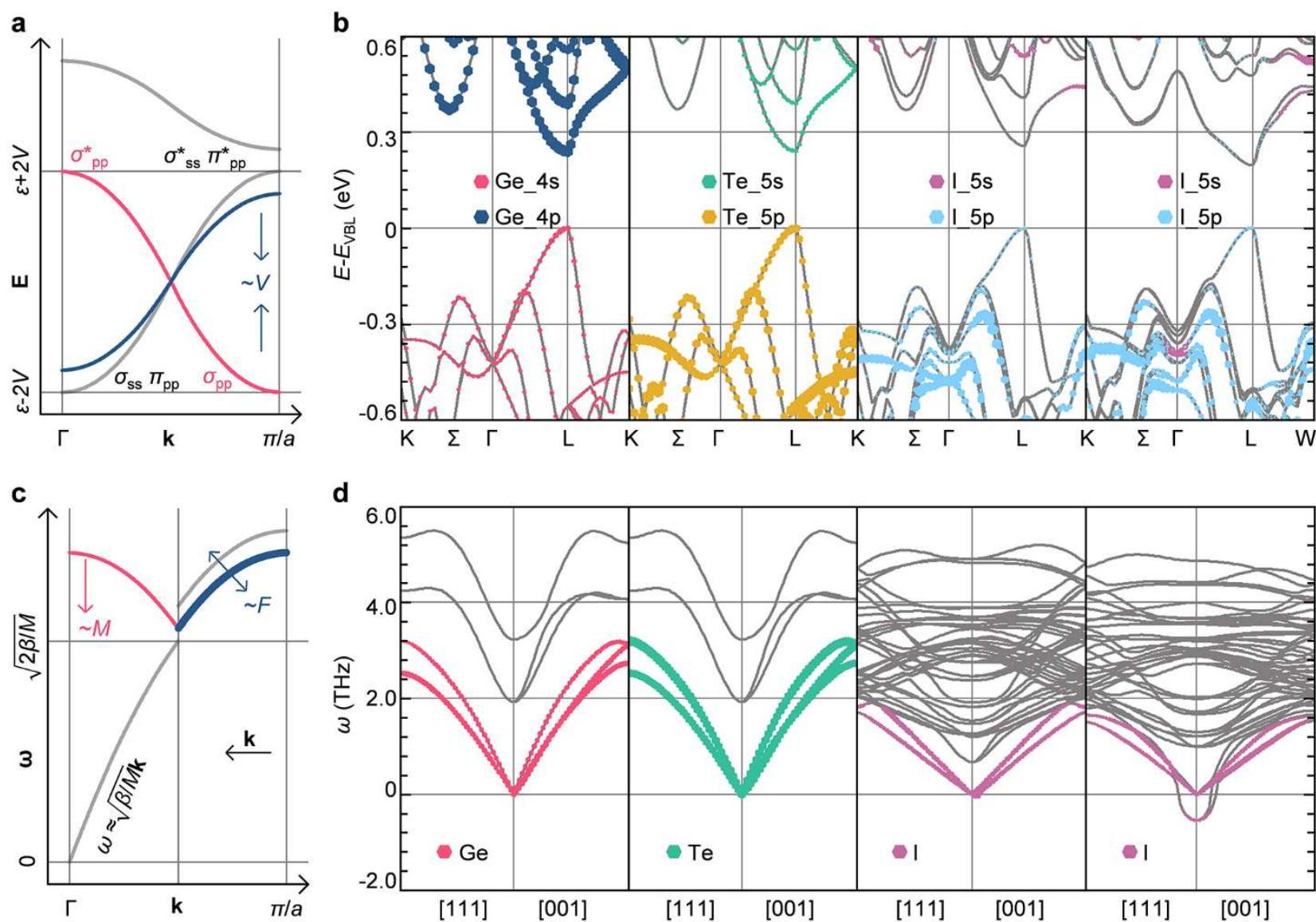

d

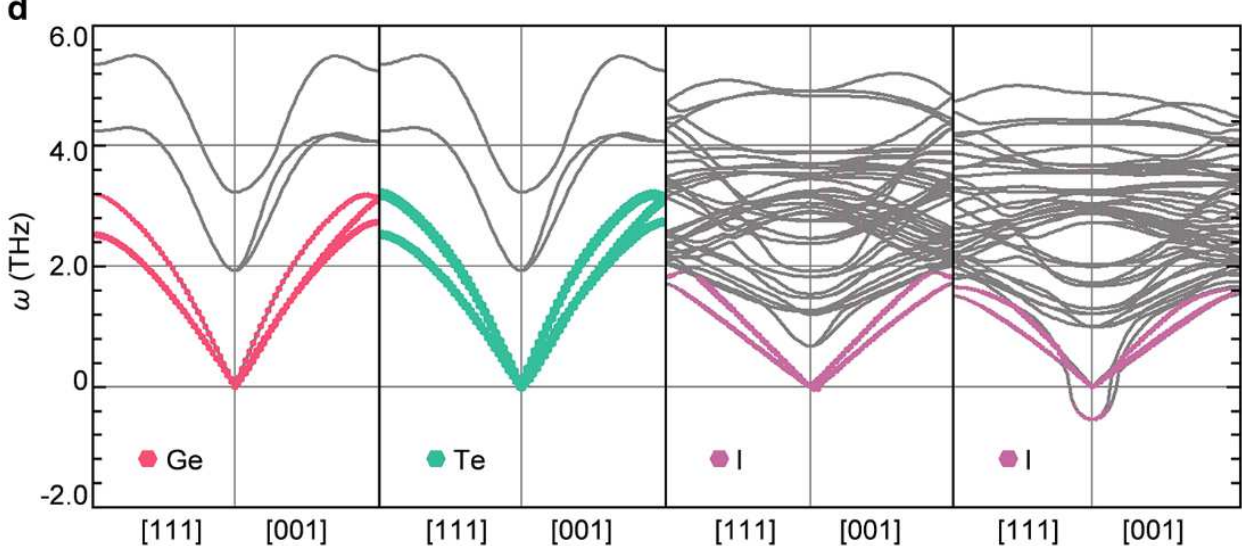

Fig. 2 Carrier and phonon transport. a, Hypothetic electron dispersion for a 1D diatomic chain with neglectable ionicity difference between two adjacent atoms. b, Calculated electron band structures based on a $3 \times 3 \times 3 \mathrm{GeTe}$ parent supercell, from left to right are $\mathrm{Ge}_{27} \mathrm{Te}_{27}, \mathrm{Ge}_{27} \mathrm{Te}_{27}, \mathrm{Ge}_{27} \mathrm{Te}_{26} \mathrm{I}_{1}$, and $\mathrm{Ge}_{26} \mathrm{Bi}_{1} \mathrm{Te}_{26} \mathrm{I}_{1}$, where the band fatness is proportional to orbital weight. c, Hypothetic phonon dispersion for a $1 \mathrm{D}$ diatomic chain with small atomic mass difference between two adjacent atoms. d, Calculated phonon band structures based on a $2 \times 2 \times 2$ GeTe parent supercell, from left to right are $\mathrm{Ge}_{8} \mathrm{Te}_{8}, \mathrm{Ge}_{8} \mathrm{Te}_{8}, \mathrm{Ge}_{8} \mathrm{Te}_{7} \mathrm{I}_{1}$, and $\mathrm{Ge}_{7} \mathrm{Bi}_{1} \mathrm{Te}_{7} \mathrm{I}_{1}$, where the band fatness is proportional to atomic displacement. The small and large imaginary frequencies $(\omega)$ in $\mathrm{Ge}_{27} \mathrm{Te}_{26} \mathrm{I}_{1}$ and $\mathrm{Ge}_{26} \mathrm{Bi}_{1} \mathrm{Te}_{26} \mathrm{I}_{1}$ are mainly ascribed to local dipole formed by anion doping of Te.

For a more complicated 3D lattice, its electron band structure can be calculated based on densityfunctional-theory (DFT, Fig. S9-11). Fig. 2b projects the constituent orbitals of Ge, Te, and I to the band structures $\mathrm{Ge}_{27} \mathrm{Te}_{27}, \mathrm{Ge}_{27} \mathrm{Te}_{26} \mathrm{I}_{1}$ and $\mathrm{Ge}_{26} \mathrm{Bi}_{1} \mathrm{Te}_{26} \mathrm{I}_{1}$, with the fatness being proportional to orbital weight. In pure GeTe, a direct band gap $\left(E_{\mathrm{g}}\right)$ opens at $\mathrm{L}(\pi / \mathrm{a}, \pi / \mathrm{a}, \pi / \mathrm{a})$ point between fully occupied valance band maximum $\left(\mathrm{VB}_{\mathrm{L}}\right)$ and vacant conduction band minimum $\left(\mathrm{CB}_{\mathrm{L}}\right)$, which are dominated by Te_5 $p^{4}$ and Ge_4 $p^{2}$ orbitals, respectively. The $n$ of GeTe is dependent on the relative position of 
Fermi potential $\left(E_{\mathrm{F}}\right)$ to $\mathrm{VB}_{\mathrm{L}}$, and can be optimized by doping Te with elements that has more $\mathrm{p}$ valence electrons or higher p orbital on-site energy, which explains the elevated $E_{\mathrm{F}}$ from $5.517 \mathrm{eV}$ in $\mathrm{Ge}_{27} \mathrm{Te}_{27}$ to $5.594 \mathrm{eV}$ in $\mathrm{Ge}_{27} \mathrm{Te}_{26} \mathrm{I}_{1}$, and from $5.067 \mathrm{eV}$ in $\mathrm{Ge}_{26} \mathrm{Te}_{27}$ to $5.110 \mathrm{eV}$ in $\mathrm{Ge}_{26} \mathrm{Te}_{26} \mathrm{I}_{1}$. Valleytronics is another factor affecting carrier transport. For instance, a convergence of $\operatorname{VB}_{\mathrm{L}}$ and $\operatorname{VB}_{\Sigma}(\sim \pi / \mathrm{a}, \sim \pi / \mathrm{a}, 0)$ by reducing their energy offset $(\Delta E)$ increases band degeneracy $\left(N_{\mathrm{v}}=4+12=16\right)$, which can be promoted by doping Te with elements for weaker s-p splitting, given that Te_5 $\mathrm{p}^{4}$ orbitals are more hybridized with $\mathrm{s}^{2}$ lone pairs at $\mathrm{VB}_{\mathrm{L}}$ than $\mathrm{VB}_{\Sigma}{ }^{22}$. This is consistent with the reduced $\Delta E$ from 0.217 $\mathrm{eV}$ in $\mathrm{Ge}_{27} \mathrm{Te}_{27}$ to $0.181 \mathrm{eV}$ in $\mathrm{Ge}_{27} \mathrm{Te}_{26} \mathrm{I}_{1}$ and $0.193 \mathrm{eV}$ in $\mathrm{Ge}_{26} \mathrm{Bi}_{1} \mathrm{Te}_{26} \mathrm{I}_{1}$.

Fig. 2c plots phonon wavefunction in the first Brillouin of a simplified 1D diatomic chain, where the mass of two adjacent atoms is closed with each other, resulting in small optical-acoustic gap. Under a harmonic presumption, phonon frequency $(\omega)$ from $L$ to $\Gamma$ point can be expressed as ${ }^{23}$ :

$$
\omega=2 \sqrt{2 F / \bar{M}} \cdot \sin \left(\frac{\pi}{2} \cdot \frac{\mathbf{k}}{\mathbf{k}_{\mathbf{c}}}\right)
$$

where $F$ and $\bar{M}$ are force constant and average atomic mass, respectively, and $\mathbf{k}_{\mathbf{c}}$ is cut-off wave vector. The $\kappa$ l of an isotropic solid, i.e., polycrystalline GeTe, is proportional to phonon velocity ( $v$, proportional to the slope of $\omega$ versus $\mathbf{k})$ and mean-free-path ( $l$, inversely proportional to optical and acoustic phonon scattering). Therefore, a low $\kappa$ prefers either a large $\bar{M}$ and low $v$, which can be realized by doping Te with heavier elements; or a small $F$ and short $l$, which is relating to doping induced lattice defects and strain, hence is sensitive to doping concentration. Moreover, some discordant doping can greatly strengthen an anharmonic effect ${ }^{24}$, which on the one hand has an equivalent influence of flattening the dispersion ${ }^{25}$ to lower $v$, on the other hand can reduce the opticalacoustic band gap to shorten $l^{26}$, usually resulting in ultralow $\kappa$ l.

We also calculated phonon band structures based on a $2 \times 2 \times 2$ parent supercell substituted with cation and/or anion dopant atoms, as shown in Fig. S12. Fig. 2d projects the atomic displacement of Ge, Te, and $\mathrm{I}$ to the band structures of $\mathrm{Ge}_{8} \mathrm{Te}_{8}, \mathrm{Ge}_{8} \mathrm{Te}_{7} \mathrm{I}_{1}$, and $\mathrm{Ge}_{7} \mathrm{Bi}_{1} \mathrm{Te}_{7} \mathrm{I}_{1}$, with the fatness being proportional 
to displacement amplitude. In pure GeTe, anion Te mainly contributes to acoustic phonons due to heavier atomic mass than Ge, which determines $\kappa_{1}$ in a wide temperature range. At $\Gamma$ point, one longitudinal (LA) and two transverse (TA) acoustic branches are triply degenerated, with an overall $\omega$ decreased from $5.9 \mathrm{THz}$ in $\mathrm{Ge}_{8} \mathrm{Te}_{8}$ to $5.1 \mathrm{THz}$ in $\mathrm{Ge}_{8} \mathrm{Te}_{7} \mathrm{I}_{1}$, then to $4.9 \mathrm{THz}$ in $\mathrm{Ge}_{7} \mathrm{Bi}_{1} \mathrm{Te}_{7} \mathrm{I}_{1}$, leading to a decreased $v$ thus $\kappa$ mainly by doping I. Meanwhile, a slight imaginary $\omega$ can be observed in $\mathrm{Ge}_{8} \mathrm{Te}_{7} \mathrm{I}_{1}$, which increases to $\sim 0.5 \mathrm{i} \mathrm{THz}$ in $\mathrm{Ge}_{7} \mathrm{Bi}_{1} \mathrm{Te}_{7} \mathrm{I}_{1}$, implying that doping I (compared to all real $\omega$ in $\left.\mathrm{Ge}_{7} \mathrm{Bi}_{1} \mathrm{Te}_{8}\right)$ increases anharmonicity by intensifying the local dipole ${ }^{27}$, giving rise to phonon scattering rates, which shortens $l$ to further decrease $\kappa$ l.

\section{Electrical and Thermal Properties}

Fig. 3a-b and Fig. S13-17 show measured temperature-dependent $\sigma$ and $S$ of pure, cation $\mathrm{Sb} / \mathrm{Bi}$ and anion $\mathrm{Br} / \mathrm{I}$ doped, and (Bi, I) co-doped GeTe, with the uncertainties estimated by propagation law (Note S5, Fig. S18, and Table S2). All above aliovalent dopants serve a donor effect to reduce $n$ (Fig. $\mathrm{S} 19)$, making $\sigma$ decreases with increasing the doping concentration, down to $100 \mathrm{~S} \cdot \mathrm{cm}^{-1}$ in $8 \%(\mathrm{Bi}, \mathrm{I})$ co-doped GeTe at $300 \mathrm{~K}$. By increasing the temperature, $\sigma$ show a metallic downward tendency, in contrast to the upward tendency of $S$, leading to an improved electronic quality factor as revealed by the plot of $S$ versus logarithmic $\sigma$ which has a slope matched with $k_{\mathrm{B}} / e$ (Fig. S20) ${ }^{28}$. A desirable $S$ of $220 \mu \mathrm{V} \cdot \mathrm{K}^{-1}$ at $700 \mathrm{~K}$ is obtained in both $\mathrm{Sb} / \mathrm{Bi}$ and I doped GeTe; however, at room temperature, $\mathrm{Sb} / \mathrm{Bi}$ doped GeTe maintains an $S$ as large as $150 \mu \mathrm{V} \cdot \mathrm{K}^{-1}$, which is only $70 \mu \mathrm{V} \cdot \mathrm{K}^{-1}$ for $\mathrm{Br} / \mathrm{I}$ doped GeTe, mainly due to cation dopants can more effectively modulate $n$ in $\alpha-\mathrm{GeTe}^{29}$. Combining the beneficial effects of both cation and anion doping, (Bi, I) co-doped GeTe show a high $S^{2} \sigma$ of $\sim 40$ $\mu \mathrm{W} \cdot \mathrm{cm}^{-1} \cdot \mathrm{K}^{-1}$ at $700 \mathrm{~K}$, which is comparable to that of pure GeTe. 

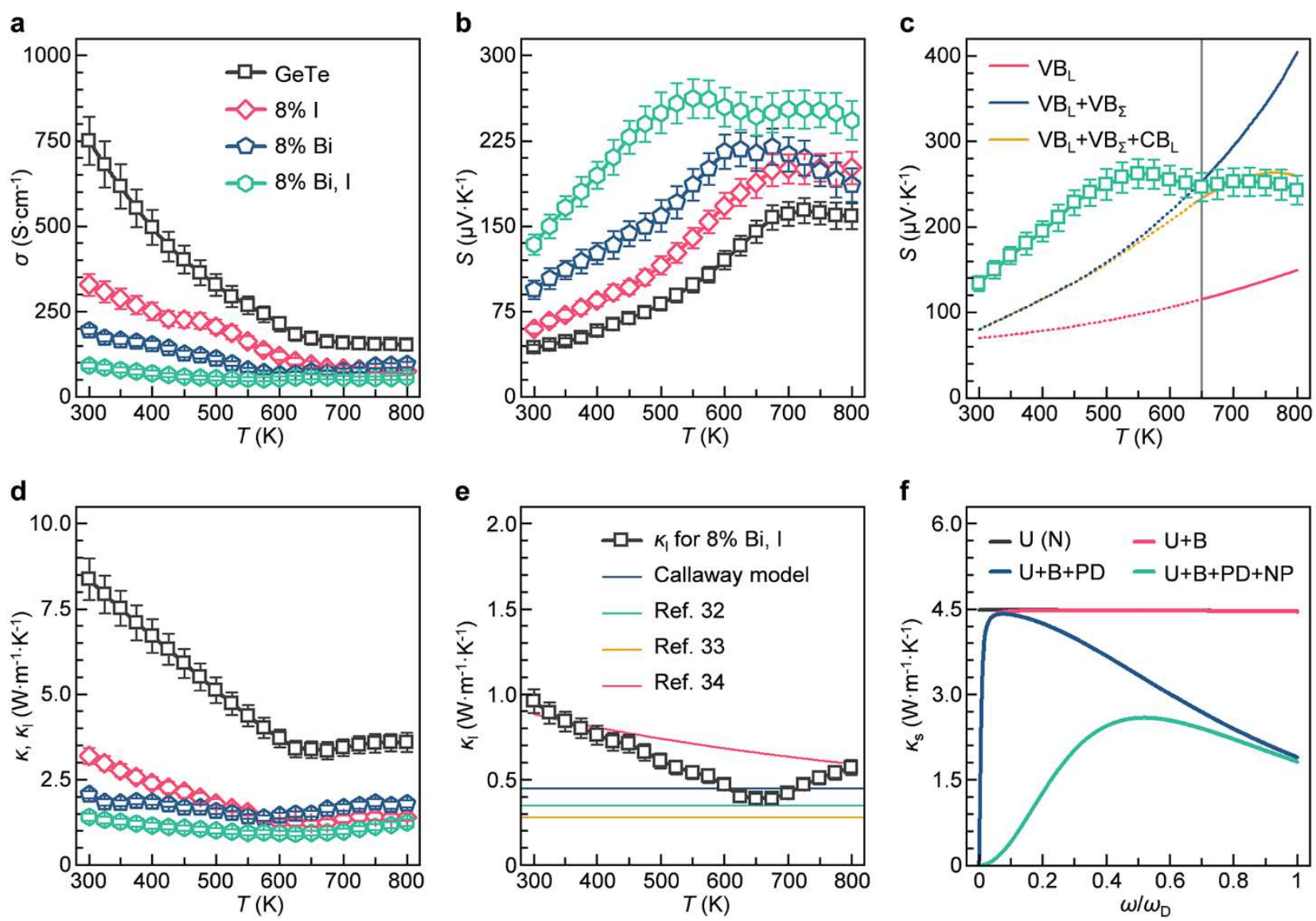

Fig. 3 Electrical and thermal properties. a-b, Change of electrical conductivity $(\sigma)$ and Seebeck coefficient $(S)$ with increasing temperature $(T)$, respectively. c, Comparison of measured $S$ of $8 \%(\mathrm{Bi}, \mathrm{I})$ co-doped GeTe with modelled $S$ based on the band structure of $\mathrm{Ge}_{26} \mathrm{Bi}_{1} \mathrm{Te}_{26} \mathrm{I}_{1}$. The modelling considers the additional contribution of light conduction band $\left(\mathrm{VB}_{\mathrm{L}}\right)$, heavy conduction band $\left(\mathrm{VB}_{\Sigma}\right)$, and valence band $\left(\mathrm{CB}_{\mathrm{L}}\right)$, to carrier transport, named as $\mathrm{VB}_{\mathrm{L}}, \mathrm{VB}_{\mathrm{L}}+\mathrm{VB}_{\Sigma}$, and $\mathrm{VB}_{\mathrm{L}}+\mathrm{VB}_{\Sigma}+\mathrm{CB}_{\mathrm{L}}$. d-e, Change of total thermal conductivity $(\kappa)$ and lattice thermal conductivity $\left(\kappa_{1}\right)$ with increasing temperature $T$, respectively. The $\kappa_{1}$ of $8 \%(\mathrm{Bi}, \mathrm{I})$ co-doped GeTe is compared with several conceptional inferior limits of lattice thermal conductance. f, Fitting $\kappa_{1}$ of $8 \%$ (Bi, I) co-doped GeTe at 300 $\mathrm{K}$ with the additional spectral lattice thermal conductivity $\left(\kappa_{\mathrm{s}}\right)$ due to different phonon scattering processes.

The optimized electrical properties can be rationalized using numerical modelling based on the Boltzmann equation. For instance, in pure GeTe, $S$ is primarily determined by $\mathrm{VB}_{\mathrm{L}}$ due to large $\Delta E$ and $E_{\mathrm{g}}$; whereas in $\mathrm{Bi}$ or I doped, and $(\mathrm{Bi}, \mathrm{I})$ co-doped $\mathrm{GeTe}$, both $\Delta E$ and $E_{\mathrm{g}}$ are decreased in accordance with doping concentration, making $\mathrm{VB}_{\Sigma}$ and $\mathrm{CB}_{\mathrm{L}}$ gradually contribute to $S$ by:

$$
S=\frac{S_{\mathrm{VBL}} \sigma_{\mathrm{VBL}}+S_{\mathrm{VB \Sigma}} \sigma_{\mathrm{VB \Sigma}}-S_{\mathrm{CBL}} \sigma_{\mathrm{CBL}}}{\sigma_{\mathrm{VBL}}+\sigma_{\mathrm{VB \Sigma}}+\sigma_{\mathrm{CBL}}}
$$


As $\mathrm{VB}_{\Sigma}$ is beneath $\mathrm{VB}_{\mathrm{L}}$, it can be regarded as degenerated band edge with a parabolic dispersion. In contrast, $\mathrm{VB}_{\mathrm{L}}$ and $\mathrm{CB}_{\mathrm{L}}$ are adjacent to $E_{\mathrm{g}}$, thus with a nonparabolic Kane dispersion ${ }^{30}$ :

$$
E\left(1+E / E_{\mathrm{g}}\right)=\frac{\hbar^{2} \mathrm{k}^{2}}{2 m^{*}}
$$

where $\hbar$ is reduced Planck constant, $m^{*}$ is dependent on $E_{\mathrm{g}}$ (and temperature). Fig. S21 shows the plot of $S$ versus $n$ at $700 \mathrm{~K}$, considering the addition of $\mathrm{VB}_{\Sigma}$ and $\mathrm{CB}_{\mathrm{L}}$ (Note S6). It indicates that the $S$ of pure and lightly doped GeTe are mainly determined by $\mathrm{VB}_{\mathrm{L}}$. By increasing the doping concentration, the plot behaves an upward tendency towards $\mathrm{VB}_{\mathrm{L}}+\mathrm{VB} \Sigma$, mainly due to the increased $N_{\mathrm{v}}$ by band convergence. As has been mentioned, doping Bi and/or I in GeTe will decrease $E_{\mathrm{g}}$ and possibly lead to a bipolar effect, making the plot of heavily $(\mathrm{Bi}, \mathrm{I})$ co-doped GeTe saturates at $\mathrm{VB}_{\mathrm{L}}+\mathrm{VB}_{\Sigma}+\mathrm{CB}_{\mathrm{L}}$. This is confirmed by Fig. 3c, where the measured and modelled $S$ of $8 \%$ (Bi, I) co-doped GeTe are well fitted in $\beta$-GeTe temperature.

Likewise, the measured temperature-dependent $\kappa$ is presented in Fig. 3d, which behave a similar tendency with $\sigma$, indicating a nonnegligible contribution of $\kappa_{\mathrm{e}}$ to $\kappa$. In addition, $\kappa$ is decreased with increasing doping concentration, down to $0.94 \mathrm{~W} \cdot \mathrm{m}^{-1} \cdot \mathrm{K}^{-1}$ in $8 \%(\mathrm{Bi}, \mathrm{I})$ co-doped GeTe, which is attributed to both decreased $\kappa_{\mathrm{e}}$ and $\kappa_{\mathrm{l}}$. We further calculated the $\kappa_{1}$ quantitatively by subtracting $\kappa_{\mathrm{e}}$ from $\kappa$, which is estimated based on the Wiedemann-Franz law, and the results are shown in Fig. 3e. As can be seen, $\mathrm{Sb} / \mathrm{Bi}$ doped GeTe has apparently lower $\kappa$ 1 than that of Br/I mono-doped GeTe near room temperature, due to $\mathrm{Sb}$ and $\mathrm{Bi}$ can enlarge the $\alpha$ of $\alpha$-GeTe to strengthen the long-range phonon scattering ${ }^{31}$, while in $\beta$-GeTe temperature, all $\kappa_{1}$ tends to merge. By co-doping Bi and I, the $\kappa_{1}$ is further decreased down to $0.39 \mathrm{~W} \cdot \mathrm{m}^{-1} \cdot \mathrm{K}^{-1}$ at $675 \mathrm{~K}$, in accordance with the reduced $\omega$ in $\mathrm{Ge}_{7} \mathrm{Bi}_{1} \mathrm{Te}_{7} \mathrm{I}_{1}$, which is even lower than some of the conceptional inferior limit of lattice thermal conductance ${ }^{32-34}$.

In addition to an intrinsic lower $\omega, \kappa$ can be extrinsically decreased by lattice defects as phonon scattering centers, based on the phenomenological Debye-Callaway model ${ }^{35}$ :

$$
\kappa_{1}=\frac{k_{\mathrm{B}}}{2 \pi^{2} v}\left(\frac{k_{\mathrm{B}} T}{\hbar}\right)^{3} \int_{0}^{\theta_{\mathrm{D}} / T} \tau_{\mathrm{p}}(z) \frac{z^{4} e^{z}}{\left(e^{z}-1\right)^{2}} \mathrm{~d} z
$$


where $\tau_{\mathrm{p}}, z$, and $\theta_{\mathrm{D}}$ are phonon relaxation time, reduced frequency, and the Debye temperature, respectively. According to Matthiessen's rule, $\tau_{\mathrm{p}}$ is an addition of participant phonon scattering processes ${ }^{36}$, including intrinsic Umklapp (U) and normal $(\mathrm{N})$ processes that can be extracted from phonon dispersion, and extrinsic scattering processes due to various types of lattice defects. Here, we take grain boundaries (B), nanoprecipitates (NP), and point defects (PD) into account for phonon scattering (Note S7 and Table S3). The integral spectral lattice thermal conductivity $\left(\kappa_{\mathrm{s}}\right)$ of each process is calculated by fitting the $\kappa_{1}$ of $8 \%(\mathrm{Bi}, \mathrm{I})$ co-doped GeTe at $300 \mathrm{~K}$, as shown in Fig. $3 \mathrm{f}$. The area between two adjacent curves represents the decreased magnitude $\kappa$ d due to additional phonon scattering. As can be seen, $\kappa_{1}$ is reduced throughout a full range of phonon spectrum, where B, NP, and PD take over low-, medium-, and high-frequency phonon scattering, respectively.

\section{Thermoelectric Device and Efficiency}

Owing to high $S^{2} \sigma$ and low $\kappa,(\mathrm{Bi}, \mathrm{I})$ co-doped GeTe shows an evidently enhanced $z T$ in both $\alpha$ - and $\beta$-GeTe temperature range. Specifically, the peak $z T$ is enhanced from 0.8 in pure GeTe to 2.5 in $8 \%$ (Bi, I) co-doped GeTe at $675 \mathrm{~K}$ (Fig. 4a), leading to an ultrahigh average $z T$ over 1.5 from 625 to 800 $\mathrm{K}$. The temperature-dependent $z T$ of $8 \%(\mathrm{Bi}, \mathrm{I})$ co-doped GeTe match with modelled $\mathrm{VB}_{\mathrm{L}}+\mathrm{VB}_{\Sigma}+\mathrm{CB}_{\mathrm{L}}$, as displayed in Fig. 4b, which confirms the contribution of $\mathrm{CB}_{\mathrm{L}}$. To evaluate the application of (Bi, I) co-doped GeTe, we assembled an 8-couple thermoelectric generator made of $8 \%$ (Bi, I) co-doped GeTe as p-type leg and $\mathrm{Yb}$ doped $\mathrm{CoSb}_{3}$ as n-type leg ${ }^{37}$. The legs are in a dimension of $4 \times 4 \times 6 \mathrm{~mm}$, which are alternatively weld on Ag electrodes. Ni and Ti buffer layers are used to block the diffusion from electrodes to p-type and n-type legs, respectively. The thermal stability and interfacial resistance of Ni-GeTe and Ti-CoSb 3 contact structures are tested by line scanning and elemental mapping, as shown in Fig. S22-23. 
a

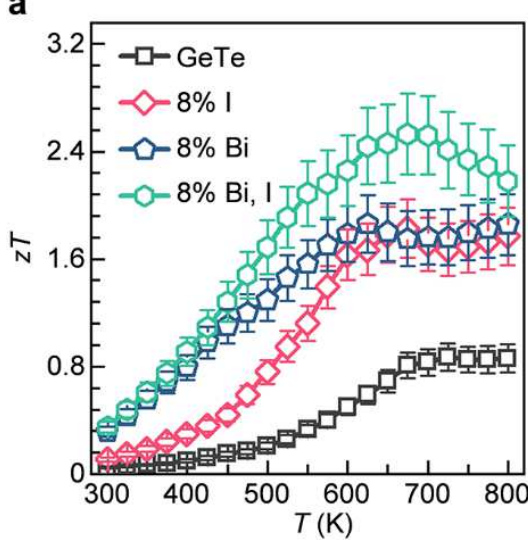

d

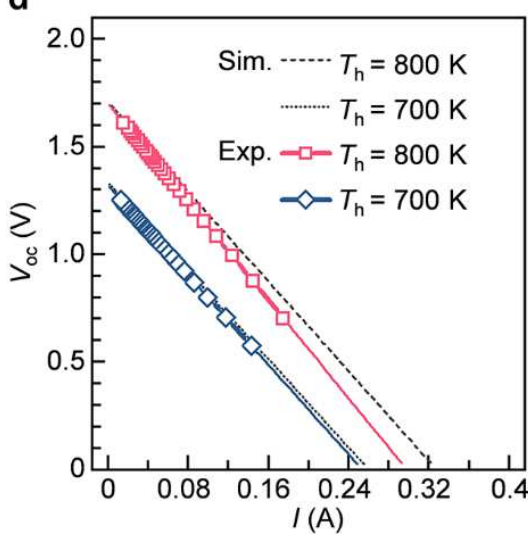

b

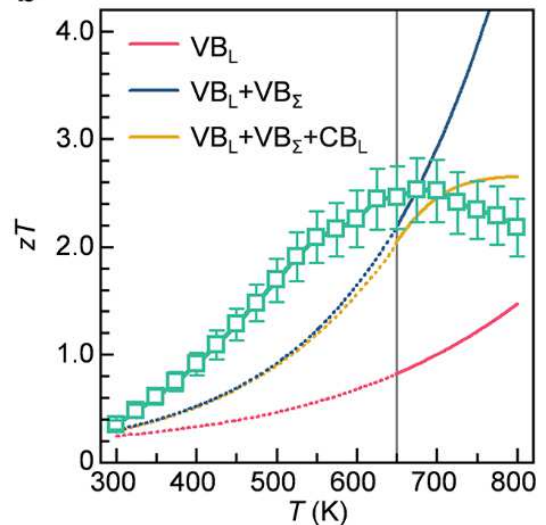

e

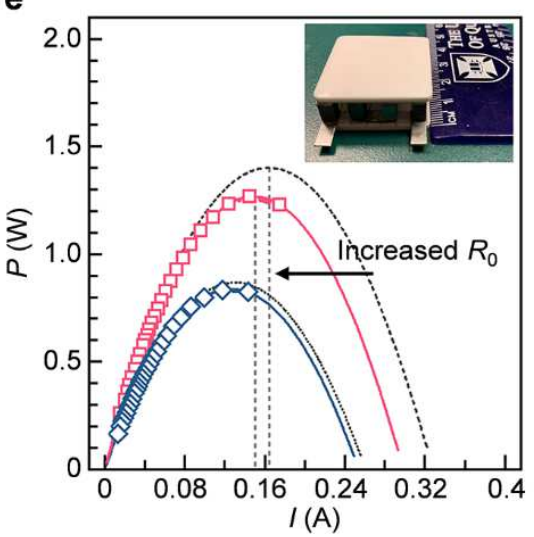

C
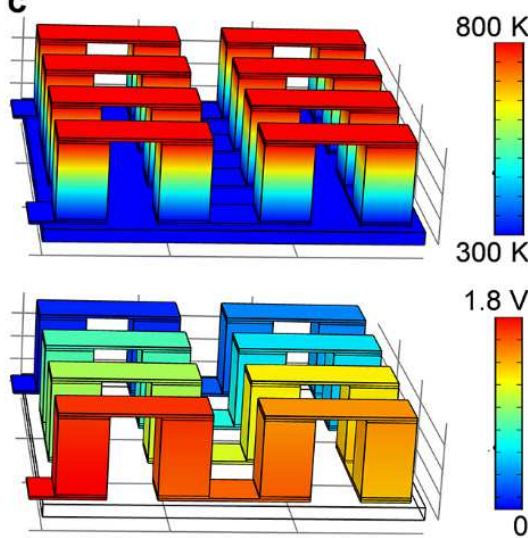

$1.8 \mathrm{~V}$

f

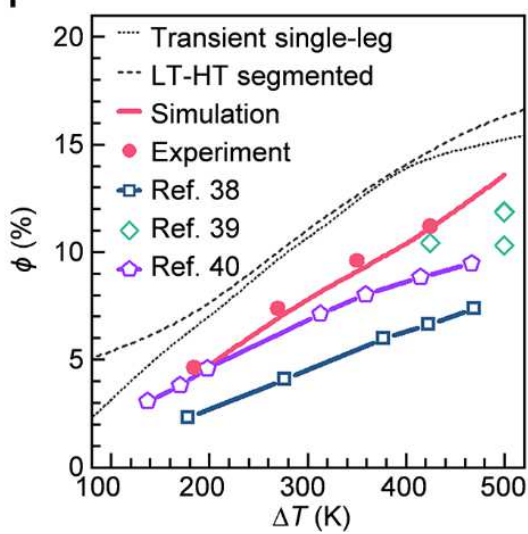

Fig. 4 Thermoelectric device and efficiency. a, Change of figure-of-merit $(z T)$ with increasing temperature $(T)$. b, Comparison of measured and modelled $z T$ of $8 \%(\mathrm{Bi}, \mathrm{I})$ co-doped GeTe. The modelling considers the additional contribution of light conduction band $\left(\mathrm{VB}_{\mathrm{L}}\right)$, heavy conduction band $\left(\mathrm{VB}_{\Sigma}\right)$, and valence band $\left(\mathrm{CB}_{\mathrm{L}}\right)$, while the lattice thermal conductivity $\left(\kappa_{1}\right)$ is supposed to be constant, named as $\mathrm{VB}_{\mathrm{L}}, \mathrm{VB}_{\mathrm{L}}+\mathrm{VB}_{\Sigma}$, and $\mathrm{VB}_{\mathrm{L}}+\mathrm{VB}_{\Sigma}+\mathrm{CB}_{\mathrm{L}}$. $\mathbf{c}$, FEA simulation of surface and contour mapping of $T$ and voltage $(V)$ of an 8-couple thermoelectric generator, with the thermal boundary condition set as cold side $T_{\mathrm{c}}=300 \mathrm{~K}$ and hot side $T_{\mathrm{h}}=800 \mathrm{~K}$. d-e, Comparison of FEA simulated and experimental open-circuit voltage $\left(V_{\mathrm{oc}}\right)$ and output power $(P)$ as a function of current $(I)$, respectively, with the thermal boundary conditions set as cold side $T_{\mathrm{c}}=300 \mathrm{~K}$ and hot side $T_{\mathrm{h}}=700$ and $800 \mathrm{~K}$. f, Conversion efficiency $(\phi)$ as a function of temperature difference $(\Delta T)$, where the $\phi$ of other GeTe-based thermoelectric generators also included for comparison.

For measurement, glass fibre was used to fill the gaps between thermoelectric legs to avoid thermal radiation. The as-assembled thermoelectric generator was compacted by alumina substrates, with the cold side contacted to a metal cooling fin frame, and the hot side controlled by a sensor for varying temperature. Fig. $4 \mathrm{c}$ and Fig. S24 show the simulated temperature and voltage profiles based on finiteelement-analysis (FEA), with input parameters including temperature-dependent $S, \sigma$, and $\kappa$ along 14 
thermoelectric legs and interfacial resistance (Table S4). The thermal boundary conditions were set as cold side $\left(T_{\mathrm{c}}\right)$ at $300 \mathrm{~K}$ and hot side $\left(T_{\mathrm{h}}\right)$ varying from 500 to $800 \mathrm{~K}$. Heat dissipation from the cold side was measured to estimate heat-to-electricity conversion.

The measured and simulated open-circuit voltage $\left(V_{\mathrm{oc}}\right)$ and output power $(P)$ as a function of current (I) are compared in Fig. 4d-e and Fig. S25. As can be seen, by increasing $T_{\mathrm{h}}$ from 500 to $800 \mathrm{~K}$, the $V_{\mathrm{oc}}$ (intercept) and internal resistance (slope) are respectively enhanced from 0.5 to $1.7 \mathrm{~V}$ and from 139 to $197 \mathrm{~m} \Omega$. $P$ shows a nearly parabolic dependence on $I$, which is proportional to temperature difference $(\Delta T)$, reaching up to $1.4 \mathrm{~W}$ under a $\Delta T$ of $500 \mathrm{~K}$. It is noticed an apparent dampening of the measured $V_{\mathrm{oc}}$ and $P$ compared to the simulated values when $T_{\mathrm{h}}$ is $800 \mathrm{~K}$, which is based on previous reports ${ }^{38}$, ascribed to the mechanical instability due to phase transition of GeTe. As illustrated in Fig. 4f, the thermoelectric generator has a simulated conversion efficiency $(\phi)$ of $13.5 \%$ and a measured $\phi$ of $11.6 \%$ under a $\Delta T$ of $500 \mathrm{~K}$, which outperforms most of the reported GeTe thermoelectric generators ${ }^{38-40}$. Such a high performance is owing to the high $z T$ value of the constituent thermoelectric materials and the rational device design. Further increase in $\phi$ can be realized by the transient measurement of a single-leg thermoelectric generator, or constructing a multi-segmented thermoelectric generator, which have been predicted in the spirit of references 41 and $42^{41,42}$.

To conclude, this work provides a comprehensive discussion on the roles of anion sites in highperformance GeTe thermoelectrics. Theoretically, we elucidate that anion and cation sites play an equivalent role in the crystal structure and bonding mechanisms of rocksalt $\beta$-GeTe, based on point group operation, molecular orbital theory, tight binding model, and DFT calculations. Experimentally, we use a modified flux-assist method to synthesize cation $\mathrm{Sb} / \mathrm{Bi}$ and anion $\mathrm{Br} / \mathrm{I}$ doped, and (Bi, I) codoped GeTe samples. Subsequent measurements indicates that $\mathrm{Sb} / \mathrm{Bi}$ and $\mathrm{Br} / \mathrm{I}$ doped $\mathrm{GeTe}$ have comparably optimized electrical and thermal properties, but due to different doping effects. Further (Bi, I) co-doped GeTe obtain an enhanced $z T$ up to 2.5 at $675 \mathrm{~K}$ and an average $z T$ over 1.5 from 625 
to $800 \mathrm{~K}$. Using p-type $8 \%(\mathrm{Bi}, \mathrm{I})$ co-doped $\mathrm{GeTe}$ and n-type $\mathrm{Yb}$ doped $\mathrm{CoSb}_{3}$, an 8-couple thermoelectric generator is assembled to yield a measured conversion efficiency of $\sim 11.6 \%$ and a simulated conversion efficiency of $\sim 13.5 \%$ under a $\Delta T$ of $500 \mathrm{~K}$. The ultrahigh material and device performance reported by this work proves the full potential of anion doping for realizing highperformance GeTe thermoelectrics.

\section{References}

1 Snyder, G. J. \& Toberer, E. S. Complex Thermoelectric Materials. Nat. Mater. 7, 105-114, (2008).

2 Shi, X. \& Chen, L. Thermoelectric Materials Step up. Nat. Mater. 15, 691-692, (2016).

3 Qin, B. et al. Momentum and Energy Multiband Alignment Enable Power Generation and Thermoelectric Cooling. Science, eabi8668, (2021).

4 Jun, M. et al. High Thermoelectric Cooling Performance of n-type Mg3Bi2-based Materials. Science 365, 495-498, (2019).

5 Jiang, B. et al. High-entropy-stabilized Chalcogenides with High Thermoelectric Performance. Science 371, 830-834, (2021).

6 Perumal, S. et al. Realization of High Thermoelectric Figure of Merit in GeTe by Complementary Co-doping of Bi and In. Joule 3, 2565-2580, (2019).

7 Luo, Y. et al. High-Performance Thermoelectrics from Cellular Nanostructured $\mathrm{Sb}_{2} \mathrm{Si}_{2} \mathrm{Te}_{6}$. Joule 4, 159-175, (2020).

8 He, J. \& Tritt, T. M. Advances in Thermoelectric Materials Research: Looking Back and Moving Forward. Science 357, eaak9997, (2017).

9 Hong, M., Zou, J. \& Chen, Z.-G. Thermoelectric GeTe with Diverse Degrees of Freedom Having Secured Superhigh Performance. Adv. Mater. 31, 1807071, (2019).

10 Pei, Y. et al. Convergence of Electronic Bands for High Performance Bulk Thermoelectrics. Nature 473, 66-69, (2011). 
11 Shportko, K. et al. Resonant Bonding in Crystalline Phase-change Materials. Nat. Mater. 7, 653$658,(2008)$.

12 Hempelmann, J. et al. Long-Range Forces in Rock-Salt-Type Tellurides and How they Mirror the Underlying Chemical Bonding. Adv. Mater. n/a, 2100163, (2021).

13 Wuttig, M., Deringer, V. L., Gonze, X., Bichara, C. \& Raty, J.-Y. Incipient Metals: Functional Materials with a Unique Bonding Mechanism. Adv. Mater. 30, 1803777, (2018).

$14 \mathrm{Li}$, M. et al. Optimizing Electronic Quality Factor toward High-Performance $\mathrm{Ge}_{1-\mathrm{x}-\mathrm{y}} \mathrm{Ta}_{\mathrm{x}} \mathrm{Sb}_{\mathrm{y}} \mathrm{Te}$ Thermoelectrics: The Role of Transition Metal Doping. Adv. Mater. n/a, 2102575, (2021).

15 Lee, S. et al. Resonant Bonding Leads to Low Lattice Thermal Conductivity. Nat. Commun. 5, $3525,(2014)$.

16 Deringer, V. L. et al. Bonding Nature of Local Structural Motifs in Amorphous GeTe. Angew. Chem. Int. Ed. 53, 10817-10820, (2014).

17 Dong, J. et al. Medium-temperature Thermoelectric GeTe: Vacancy Suppression and Band Structure Engineering Leading to High Performance. Energy Environ. Sci. 12, 1396-1403, (2019).

18 Lee, H. S. et al. Herringbone Structure in GeTe-based Thermoelectric Materials. Acta Mater. 91, 83-90, (2015).

19 He, J., Kanatzidis, M. G. \& Dravid, V. P. High Performance Bulk Thermoelectrics via a Panoscopic Approach. Mater. Today 16, 166-176, (2013).

20 Zeier, W. G. et al. Thinking Like a Chemist: Intuition in Thermoelectric Materials. Angew. Chem. Int. Ed. 55, 6826-6841, (2016).

21 Brod, M. K., Toriyama, M. Y. \& Snyder, G. J. Orbital Chemistry That Leads to High Valley Degeneracy in PbTe. Chem. Mater. 32, 9771-9779, (2020).

22 Brod, M. K. \& Snyder, G. J. Orbital Chemistry of High Valence Band Convergence and Lowdimensional Topology in PbTe. J. Mater. Chem. A 9, 12119-12139, (2021).

$23 \mathrm{Wu}$, Y. et al. Lattice Strain Advances Thermoelectrics. Joule 3, 1276-1288, (2019). 
24 Dutta, M., Biswas, R. K., Pati, S. K. \& Biswas, K. Discordant Gd and Electronic Band Flattening Synergistically Induce High Thermoelectric Performance in n-type PbTe. ACS Energy Lett. 6, 1625-1632, (2021).

25 Delaire, O. et al. Giant Anharmonic Phonon Scattering in PbTe. Nat. Mater. 10, 614-619, (2011).

26 Hong, M. et al. Strong Phonon-Phonon Interactions Securing Extraordinary Thermoelectric Ge1${ }_{x} S b_{x}$ Te with Zn-Alloying-Induced Band Alignment. J. Am. Chem. Soc. 141, 1742-1748, (2019).

27 Božin, E. S. et al. Entropically Stabilized Local Dipole Formation in Lead Chalcogenides. Science 330, 1660-1663, (2010).

28 Madar, N., Givon, T., Mogilyansky, D. \& Gelbstein, Y. High Thermoelectric Potential of Bi2 $\mathrm{Te}_{3}$ Alloyed GeTe-rich Phases. J. Appl. Phys. 120, 035102, (2016).

29 Liu, Z. et al. High Power Factor and Enhanced Thermoelectric Performance in Sc and Bi Codoped GeTe: Insights into the Hidden Role of Rhombohedral Distortion Degree. Adv. Energy Mater. 10, 2002588, (2020).

30 Kane, E. in Semiconductors and Semimetals Vol. 1, 75-100 (Elsevier, 1966).

31 Li, J. et al. Low-Symmetry Rhombohedral GeTe Thermoelectrics. Joule 2, 976-987, (2018).

32 Cahill, D. G., Watson, S. K. \& Pohl, R. O. Lower Limit to the Thermal Conductivity of Disordered Crystals. Phys. Rev. B 46, 6131-6140, (1992).

33 Clarke, D. R. Materials Selection Guidelines for Low Thermal Conductivity Thermal Barrier Coatings. Surf. Coat. Technol. 163-164, 67-74, (2003).

34 Agne, M. T., Hanus, R. \& Snyder, G. J. Minimum Thermal Conductivity in the Context of Diffuson-mediated Thermal Transport. Energy Environ. Sci. 11, 609-616, (2018).

35 Callaway, J. \& Baeyer, H. C. v. Effect of Point Imperfections on Lattice Thermal Conductivity. Phys. Rev. 120, 1149, (1960).

36 Bass, J. Deviations from Matthiessen's Rule. Adv. in Phys. 21, 431-604, (1972).

37 Wang, S. et al. High-performance n-type $\mathrm{Yb}_{\mathrm{x}} \mathrm{Co}_{4} \mathrm{Sb}_{12}$ : from Partially Filled Skutterudites towards Composite Thermoelectrics. NPG Asia Mater. 8, e285-e285, (2016). 
38 Xing, T. et al. High Efficiency GeTe-based Materials and Modules for Thermoelectric Power Generation. Energy Environ. Sci. 14, 995-1003, (2021).

39 Snyder, G. J. Application of the Compatibility Factor to the Design of Segmented and Cascaded Thermoelectric Generators. Appl. Phys. Lett. 84, 2436-2438, (2004).

40 Crane, D. T., Kossakovski, D. \& Bell, L. E. Modeling the Building Blocks of a 10\% Efficient Segmented Thermoelectric Power Generator. J. Electron. Mater. 38, 1382-1386, (2009).

$41 \mathrm{Bu}$, Z. et al. Realizing a 14\% Single-leg Thermoelectric Efficiency in GeTe Alloys. Science Adv. 7, eabf2738, (2021). 


\section{Method}

Synthesis and characterization. Polycrystalline samples were synthesized by a modified flux-assist process. First, high-purity Ge, Te, Bi, Sb (5N, Sigma-Aldrich), BiI 3 (3N, Sigma-Aldrich), TeI 4 (99.8\%, Novachem), and $\mathrm{TeBr}_{4}(97 \%$, Novachem) were weighed out based on the stoichiometry of co-doped $\mathrm{Ge}_{1-x} \mathrm{Bi}_{x} \mathrm{Te}_{1-y} \mathrm{I}_{y}$ and mono-doped $\mathrm{Ge}_{1-x} \mathrm{Sb}_{x} \mathrm{Te}, \mathrm{Ge}_{1-x} \mathrm{Bi}_{x} \mathrm{Te}, \mathrm{GeTe}_{1-y} \mathrm{Br}_{y}$, and $\mathrm{GeTe}_{1-y} \mathrm{I}_{y}(x=y=0.02 \sim 0.08)$, which were then mixed with quintuple weight of $50 \% \mathrm{NaCl}+50 \% \mathrm{KCl}$ molten salt (4N, ProSciTech) and sealed in evacuated silica tubes. Second, the quartz tubes were heated up to $700{ }^{\circ} \mathrm{C}$ for $1440 \mathrm{~min}$ and quenched in ice water, then annealed at $600{ }^{\circ} \mathrm{C}$ for $4320 \mathrm{~min}$ in a muffle furnace (HT-1700, Carbolite Gero). Third, the obtained ingots were pulverized into fine powder by high-energy ball milling (8000D Mixer/Mill $\left.{ }^{\circledR}, \mathrm{AXT}\right)$, then purified using centrifuging and dried in baking oven. Last, the collected powders were consolidated using a spark plasma sintering (SPS-211Lx, Fuji Electronic) at $500{ }^{\circ} \mathrm{C}$ under Argon atmosphere, applied with a uniaxial pressure of $55 \mathrm{MPa}$.

The phase purity of the synthesized samples was identified by X-ray diffraction (XRD, D8 Advance MKII, Bruker) equipped with monochromatized $\mathrm{Cu} \mathrm{K} \alpha$ radiation $(\lambda=1.5418 \AA)$. XRD Rietveld refinement was conducted using GSAS-II software ${ }^{43}$. The microstructures were characterized by a field-emission scanning electron microscope (SEM, JSM-7001F, JEOL) using secondary electron (SE), backscattered electron (BSE) and energy-dispersive X-ray spectroscopy (EDS) techniques. The lattice structures were investigated by an aberration-corrected transmission electron microscope (TEM, HF-5000, Hitachi), assisted with Wiener filter, average background subtraction filter, and geometric phase analysis ${ }^{44,45}$. The lamella specimens were prepared using focus ion beam (FIB) lift out technique (FEI Scios Dualbeam system, Thermo Scientific). The overall grain morphology and twin structures were characterized by electron backscattered diffraction (EBSD) coupled in the FIB system. 
Thermoelectric and transport measurement. Temperature-dependent Seebeck coefficient $(S)$ and electrical conductivity $(\sigma)$ were measured by four-probe method (ZEM-3, ULVAC-RIKO). The thermal conductivity $(\kappa)$ is calculated by:

$$
\kappa=D \times C_{\mathrm{p}} \times \rho
$$

where $D$ is thermal diffusivity measured by a laser flash method (LFA $467, \mathrm{NETZSCH),} C_{\mathrm{p}}$ is specific heat capacity measured by a differential scanning calorimeter (DSC 404 F3, NETZSCH) and fitted with Dulong-Petit's law, $\rho$ is density measured by Archimedes' method.

Hall transport properties relative to increasing temperature were measured by Van der Pauw method (homemade instrument) with a sweeping magnetic field of up to $\pm 1.5 \mathrm{~T}$. Hall carrier concentration $\left(n_{\mathrm{H}}\right)$ and Hall mobility $\left(\mu_{\mathrm{H}}\right)$ are calculated based on:

$$
\begin{gathered}
n_{\mathrm{H}}=1 /\left(e \times R_{\mathrm{H}}\right) \\
\mu_{\mathrm{H}}=\sigma \times R_{\mathrm{H}}
\end{gathered}
$$

where $R_{\mathrm{H}}$ and $e$ are Hall coefficient and electron charge, respectively.

First-principle calculations. Density-functional-theory (DFT) calculations were performed based on projector-augmented wave (PAW) method as implemented in the Vienna Ab initio Simulation Package (VASP) ${ }^{46-51}$. Relativistic Perdew-Burke-Ernzerhof generalized gradient approximation functional (GGA-PBE) was adopted to treat exchange correlation interaction ${ }^{52}$. The $3 \times 3 \times 3$ and $2 \times 2 \times 2$ supercells were built for electron and force calculations, then sampled by a $9 \times 9 \times 9$ and a $15 \times 15 \times 15$ Monkhorst-Pack k-mesh, respectively ${ }^{53}$. All supercells were fully relaxed with plane wave cut-off energy of $500 \mathrm{eV}$, and convergence criterion of $1 \times 10^{-7} \mathrm{eV}$ per electron and $1 \times 10^{-3} \mathrm{eV} \cdot \AA^{-1}$ per atom. The dispersion relations were calculated along a line-mode k-path based on Brillouin path features of AFLOW framework ${ }^{53}$. The electron band structures were calculated considering spinorbital-coupling (SOC) effect. The phonon band structures were calculated without considering the SOC effect, by solving the harmonic force constant near $\Gamma$ point using Phonopy package ${ }^{54}$. 
Device fabrication and FEA simulation. Finite element analysis (FEA) simulations were performed on COMSOL Multiphysics software. An 8-couple thermoelectric generator was modelled and meshed, which were consisted of $\mathrm{Al}_{2} \mathrm{O}_{3}$ substrates, Ag electrodes, Ni or Ti buffer layers, and p-type and n-type thermoelectric legs ${ }^{42}$. The temperature-dependent $S, \sigma$ and $\kappa$ of constituent materials, and interfacial contact resistance were input to the system ${ }^{38,55,56}$. The thermal boundary condition was set as cold side at constant $300 \mathrm{~K}$, while the hot side varying with increasing temperature, which generates an output power $(P)$ by:

$$
P=V \times I
$$

where $V$ and $I$ are voltage and current, respectively. The heat dissipation $\left(Q_{\text {out }}\right)$ from cold side was estimated for calculating the heat-to-electricity efficiency $(\phi)$, namely:

$$
\begin{gathered}
Q_{\text {out }}=q \times A \\
\phi=P /\left(P+Q_{\text {out }}\right)
\end{gathered}
$$

where $q$ and $A$ are heat flux density and cross-section of thermoelectric legs, respectively.

We assembled an 8-couple thermoelectric generator to examine the simulation results. To make a ptype leg, first, the Ni-Ge ${ }_{0.92} \mathrm{Bi}_{0.08} \mathrm{Te}_{0.92} \mathrm{I}_{0.08}-\mathrm{Ni}$ powders were isostatically pressed; second, the pressed bulks were sintered using SPS under $450{ }^{\circ} \mathrm{C}$ and $30 \mathrm{MPa}$; third, the sintered pellets were cut into $4 \times 4 \times 6 \mathrm{~mm}$ rectangular bars. Likewise, the Ti-Ybo.075 $\mathrm{CoSb}_{3}$-Ti powders were made into n-type legs 37. The alternative p-type and n-type legs were soldered on Ag plates at both hot and cold sides with tin-based solder, then compacted by two $\mathrm{Al}_{2} \mathrm{O}_{3}$ substrates. Glass fibers were used to further fill the gaps between the legs to prohibit thermal radiation.

To test the thermal stability between thermoelectric materials and buffer layers, both Ni-GeTe and $\mathrm{Ti}-\mathrm{CoSb}_{3}$ contact structures were sealed in evacuated quartz tubes and soaked at $700 \mathrm{~K}$ for up to 4 days. After that, EDS mapping and line scanning were used to determine the inter-diffusion and resistance through the interfaces. The performance of as-assembled thermoelectric generator was measured by a home-made testing system under Argon atmosphere, where the cold side was 
connected to a metal fin frame, and the hot side is controlled by a temperature sensor with varying temperature from 500 to $800 \mathrm{~K}$.

\section{Data availability}

All data generated or analysed during this study are included in this article and the Supplementary Information.

\section{References}

42 Hong, M. et al. Computer-aided Design of High-efficiency GeTe-based Thermoelectric Devices. Energy Environ. Sci. 13, 1856-1864, (2020).

43 Toby, B. H. \& Dreele, R. B. V. GSAS-II: the Genesis of a Modern Open-source All Purpose Crystallography Software Package. J. Appl. Cryst. 46, 544-549, (2013).

44 Kilaas, R. Optimal and Near-optimal Filters in High-resolution Electron Microscopy. J. Microsc. 190, 45-51, (1998).

45 Hÿtch, M. J., Snoeck, E. \& Kilaas, R. Quantitative Measurement of Displacement and Strain Fields from HREM Micrographs. Ultramicroscopy 74, 131-146, (1998).

46 Kresse, G. \& Hafner, J. Ab Initio Molecular-dynamics Simulation of the Liquid-metalamorphous-semiconductor Transition in Germanium. Phys. Rev. B 49, 14251-14269, (1994).

47 Kresse, G. \& Hafner, J. Ab Initio Molecular Dynamics for Liquid Metals. Phys. Rev. B 47, 558561, (1993).

48 Kresse, G. \& Furthmüller, J. Efficiency of Ab-initio Total Energy Calculations for Metals and Semiconductors Using a Plane-wave Basis Set. Comput. Mater. Sci. 6, 15-50, (1996).

49 Kresse, G. \& Hafner, J. Norm-conserving and Ultrasoft Pseudopotentials for First-row and Transition Elements. J. Phys. Condens. Matter 6, 8245-8257, (1994).

50 Kresse, G. \& Furthmüller, J. Efficient Iterative Schemes for Ab Initio Total-energy Calculations Using a Plane-wave Basis Set. Phys. Rev. B 54, 11169-11186, (1996). 
51 Kresse, G. \& Joubert, D. From Ultrasoft Pseudopotentials to the Projector Augmented-wave Method. Phys. Rev. B 59, 1758-1775, (1999).

52 Perdew, J. P., Burke, K. \& Ernzerhof, M. Generalized Gradient Approximation Made Simple. Phys. Rev. Lett. 77, 3865-3868, (1996).

53 Setyawan, W. \& Curtarolo, S. High-throughput Electronic Band Structure Calculations: Challenges and Tools. Comput. Mater. Sci. 49, 299-312, (2010).

54 Togo, A. \& Tanaka, I. First Principles Phonon Calculations in Materials Science. Scr. Mater. 108, 1-5, (2015).

55 Zhang, Q. et al. Realizing a Thermoelectric Conversion Efficiency of $12 \%$ in Bismuth Telluride/Skutterudite Segmented Modules through Full-parameter Optimization and Energyloss Minimized Integration. Energy Environ. Sci. 10, 956-963, (2017).

56 Xing, Y. et al. High-efficiency Half-Heusler Thermoelectric Modules Enabled by Selfpropagating Synthesis and Topologic Structure Optimization. Energy Environ. Sci. 12, 33903399, (2019). 


\section{Acknowledgements}

The authors acknowledge financial support provided by the Australia Research Council and the USQ Strategic research fund. Centre for Microscopy and Microanalysis in the University of Queensland, and Australian Microscopy and Microanalysis Research Facility is acknowledged for providing the

characterization facilities. Research Computing Centre in the University of Queensland and National Computational Infrastructure supported by the Australian Government for computation resources and services is acknowledged for providing computing facilities. The authors acknowledge Dr. J.X. Liu, Dr. Y. Yin, and A.Prof. T.L. Feng for technical support; Dr. W.Y. Zhao, Dr. Q.Y. Tan, and Dr. D.L. Cortie for discussion; and Prof. P.A. Thrower for instruction on manuscript writing.

\section{Author contributions}

M.L. and Z.G.C. proposed the conception of this work and design relevant experiments. M.L. and M.H. conducted DFT calculations and FEA simulations. M.L., S.D.X., W.Y.L. and Y.W. contributed to the experimental work. Z.G.C. supervised the project. M.L., Z.G.C., H.M.C., M.D. and J.Z. drafted the manuscript. All authors have read and edited the manuscript.

\section{Competing interests}

The authors declare no competing interests.

\section{Additional information}

Supplementary information The online version contains supplementary material available at xxx.

Correspondence and requests for materials should be addressed to Zhi-Gang Chen.

Peer review information Nature Energy thanks xxx.

Reprints and permissions information is available at www.nature.com/reprints. 


\section{Supplementary Files}

This is a list of supplementary files associated with this preprint. Click to download.

- supportinginformation.docx 